\title{
Dexmedetomidina en el bloqueo del plano transverso del abdomen
}

\section{Dexmedetomidine in transverse abdominal plane block}

\begin{abstract}
Josefina Altamira García ${ }^{a}$, Rodrigo César León Hernández ${ }^{b}$ y Hortensia Castañeda Hidalgo ${ }^{c}$ Abstract:

Acute pain has been reported by $>80 \%$ in postoperative patients and is considered severe and extreme in $39 \%$ of patients. Dexmedetomidine is a drug used in intensive therapy and anesthetic practice to achieve adequate sedation and analgesia in adult and pediatric populations in a variety of surgical interventions and during the perioperative period, therefore the objective of this paper is to describe the current state of dexmedetomidine management in postoperative analgesia. From the review of specialized literature, it is possible to conclude that the combined use of dexmedetomidine in blocking the transverse abdominal plane shortens the postoperative recovery term, thereby decreasing the hospital stay and the potential risk of nosocomial infections.
\end{abstract}

Keywords:

Dexmedetomidine, postoperative pain, blockage of the transverse abdominal

\section{Resumen:}

El dolor agudo ha sido reportado por $>80 \%$ en pacientes postoperados y es considerado como severo y extremo en un $39 \%$ de los pacientes. La dexmedetomidina es un fármaco comúnmente empleado en la terapia intensiva y en la práctica anestésica para lograr una adecuada sedación y analgesia en poblaciones de adultos y pediátricos en una variedad de intervenciones quirúrgicas y durante el perioperatorio, por lo tanto el presente ensayo tiene como objetivo describir el estado actual del manejo de dexmedetomidina en analgesia postoperatoria. A partir de la revisión de la literatura especializada se concluye que el uso combinado de dexmedetomidina en el bloqueo del plano transverso abdominal acorta el período de recuperación posquirúrgica disminuyendo con ello la estancia intrahospitalaria y el riesgo potencial de infecciones intranosocomiales.

\section{Palabras Clave:}

Dexmedetomidina, dolor postoperatorio, bloqueo del plano transverso del abdomen

\section{Introducción}

La conceptualización del dolor hapresentado variaciones, en una de sus primeras definiciones, realizada por la Asociación Internacional para el Estudio del Dolor (IASP) en 1979, se le concibe como "una experiencia sensorial y emocional desagradable, asociada a un daño tisular real o potencial o descrita en términos de dicho daño". Esta misma asociación define al dolor agudo como un dolor de reciente inicio, de duración limitada; generalmente tiene una relación causal o temporal identificable, la cual se relaciona con una lesión o enfermedad. Se caracteriza por presentar máxima intensidad en las primeras 24 horas. $^{2}$
Se ha documentado que más del $80 \%$ de los pacientes sometidos a procedimientos quirúrgicos experimentaron dolor agudo postoperatorio; aproximadamente el $75 \%$ reportaron intensidad del dolor de moderada a severa o extrema. ${ }^{3}$ La evidencia sugiere que menos de la mitad de los pacientes reportan un adecuado alivio del dolor postoperatorio. Además, se conoce que el control inadecuado del dolor afecta negativamente la calidad de vida, la función, su recuperación, el riesgo de complicaciones posquirúrgicas y el riesgo de dolor persistente postquirúrgico. ${ }^{4}$

\footnotetext{
a Departamento de Anestesiología Hospital regional de Cd. Madero Pemex, División de estudios de posgrado Universidad Autónoma de Tamaulipas, https://orcid.org/0000-0001-5486-1437,Email: josealtamir@hotmail.com

b Autor de Correspondencia, Catedrático Consejo Nacional de Ciencia y Tecnología, https://orcid.org/0000-0001-5780-4382, Email: rcleonhe@ conacyt.mx
} 
El tratamiento del dolor agudo postoperatorio es multimodal ya que se genera un efecto dependiente y sinérgico entre los diferentes fármacos y técnicas utilizadas con diferentes mecanismos de acción para mejorar el control del dolor y disminuir los efectos secundarios relacionados con la utilización de algún grupo farmacológico como los opioides. Entre los fármacos utilizados para el control del dolor postoperatorio destacan los analgésicos antiinflamatorios no esteroideos, anestésicos locales, gabapentinoides, agonistas adrenérgicos $\alpha-2$, fenciclidinas y paracetamol. Cualquier combinación de estas terapias ayuda a reducir el estrés quirúrgico y mejorar los resultados en el control del dolor. ${ }^{5}$

De las técnicas regionales, se considera al boqueo del plano transverso abdominal (TAP) como una técnica de anestesia regional que bloquea nervios aferentes anterolaterales de la pared abdominal. Los tres músculos en la región anterolateral asociados con el bloqueo TAP son el oblicuo externo, el oblicuo interno, y el músculo transverso del abdomen. Esta región (piel, músculos, y peritoneo parietal) son inervados por las ramas anteriores de los nervios espinales de T7 - L1. intercostal (T9 - T11), subcostal (T12), ilioinguinal (L1), e iliohipogástrico (T12 L1), estos nervios viajan a través del espacio TAP, ubicado en un espacio entre el oblicuo interno y el mú sculo transverso del abdomen. El bloqueo puede ser realizado usando un abordaje subcostal, medioaxilar o posterior. La evidencia sugiere que la distribución del anestésico local es dependiente del abordaje utilizado. ${ }^{6}$

Debido al avance de la ultrasonografía, en los últimos años se ha motivado el desarrollo en las técnicas de anestesia y analgesia regional para el control del dolor intra y postoperatorio en pacientes adultos. El realizar bloqueos ecoguiados ha permitido mejorar la eficacia y seguridad reportando una tasa de éxito de 95-100\%. Las técnicas de bloqueo regional guiadas por ultrasonido hacen posible visualizar las estructuras nerviosas a bloquear y las referencias anatómicas en tiempo real, lo que disminuye el riesgo de punción de estructuras vecinas, o de daño directo sobre el nervio, observando la correcta distribu ción del anestésico local administrado. ${ }^{7}$

Existe evidencia mostrando la eficacia para proporcionar analgesia perioperatoria con la técnica guiada por ultrasonido. Desafortunadamente, una sola inyección de bloqueo TAP suministra analgesia de la pared abdominal solo por 8 horas, lo cual limita su uso. Diversos adyuvantes (dexmedetomidina, fentanyl, MgSO4) han sido añadidos al anestésico local para prolongar la duración analgésica del bloqueo TAP; la dexmedetomidina es un fármaco multifunción propuesto como apoyo para bloqueos regionales, pero el régimen preferible y las dosis óptimas de los adyuvantes agregados al anestésico local siguen bajo escrutinio. ${ }^{8}$ La dexmedetomidina, un potente agonista de los receptores adrenérgicos $\alpha-2$, con propiedades simpaticolíticas, sedativas, amnésicas y analgésicas, ha sido descrito como un fármaco auxiliar importante en diferentes aplicaciones clínicas. Por ejemplo, el estereoisómero de la medetomidina es utilizado en el régimen veterinario, como analgésico y sedante en animales, junto con la xilazina. ${ }^{9}$ Con base en lo anterior, el presente ensayo tiene como finalidad describir el estado actual del manejo de dexmedetomidina en analgesia postoperatoria.

La dexmedetomidina fue aprobada por la Food Drug Administration (FDA) e introducida en la práctica clínica en 1999 como sedante intravenoso de corta duración para la unidad de cuidados intensivos y tiempo después se observaron las propiedades analgésicas, por lo que se utiliza como adyuvante en la terapia analgésica sistémica y regional. ${ }^{10,11}$

\section{Receptores adrenérgicos}

El receptor adrenérgico $\alpha-2$ está constituido de tres isorreceptores: $\alpha-2 a, \alpha-2 b, y$, $\alpha-2 c$, que ligan agonistas y antagonistas $\alpha-2$ con afinidades similares y comparten una semejante composición de aminoácidos de aproximadamente 70 a $75 \% .^{12}$ Los receptores adrenérgicos $\alpha-2$ son transmembranales y están ligados a proteínas G, se conectan selectivamente a ligandos extracelulares que pueden ser o mediadores endógenos o moléculas exógenas, como fármacos. Cuando dichas moléculas interactúan con él, se reduce la entrada de calcio en las terminales nerviosas lo que se traduce en sedación, hipnosis, analgesia, simpaticolísis, neuroprotección e in hibición de secreción de la insulina. El agonismo con el receptor $a-2 b$ disminuye el temblor, produce analgesia en el cordón espinal y ocasiona vasoconstricción en las arterias periféricas; el receptor $\alpha$ 2c se asocia a modulación del proceso de cognición sensorial. ${ }^{13}$

La actividad $\alpha-2$ adrenérgica forma una parte primordial en la red intrínseca de control del dolor en el sistema nervioso central. Los subtipos relacionados con la analgesia son los tipos $\alpha-2 a$ y $\alpha-2 c$, que se localizan en las terminales nerviosas aferentes primarias de las fibras $C$ y en algunos sitios de la médula espinal, como en la región del asta dorsal superficial; el subtipo $a-2 b$ está ubicado a nivel presináptico, postsináptico y extrasináptico, como en plaquetas, ojos, riñones, e hígado mientras que en la médula espinal existen niveles mínimos. ${ }^{12,13}$

Farmacocinética 
La estructura de la dexmedetomidina es imidazólica similar a los agonistas a-2 adrenérgicos; su nombre químico es clorhidrato de dexmedetomidina es (4- [(1S)[1-(2,3 dimetilfenil) etil]-1 $\mathrm{H}$-imidazolmonoclorhidrato. ${ }^{14} \mathrm{Su}$ fórmula química es $\mathrm{C}_{13} \mathrm{H}_{16} \mathrm{~N}_{2}$. $\mathrm{HCl}$. Su peso moleculares de 236.7.10

Absorción: aunque la dexmedetomidina está solo registrada para uso i.v, múltiples rutas de administración han sido investigadas. Cuando la administración es extravascular se pueden observar altos niveles plasmáticos en relación con los observados con la administración intravenosa. Después de la administración oral el efecto de primer paso es amplio, con una biodisponibilidad del $16 \%$. Es efectivamente absorbida por la mucosa nasal y bucal, esta característica es importante cuando se pretende utilizar en niños o pacientes geriátricos. $^{13}$

La vía transdérmica presenta una biodisponibilidad del $51 \%$, con una vida media terminal de 5.6 horas, y se observa un efecto sedante entre una a dos horas después de la administración. ${ }^{13}$ Posterior a infusión intravenosa, la dexmedetomidina tiene una fase rápida de distribución, con una vida media de 6 minutos, y una vida media de eliminación terminal de aproximadamente 2 horas con aclaramiento de 39 litros por hora. El volumen de distribución es de 118 litros aproximadamente; su vida media de eliminación terminal es de 2 horas aproximadamente. ${ }^{14}$

De manera intramuscular, la concentración plasmática máxima se alcanza de 1.6 a 1.7 horas, con una vida media de eliminación de 1.6 a 2.4 horas y un aclaramiento plasmático total de 0.7 a $0.9 \mathrm{~L} / \mathrm{h} / \mathrm{kg}$; con volumen aparente de distribución de 2.1 a $2.6 \mathrm{~L} / \mathrm{kg}$. Por otra parte, la vía subcutánea o intramuscular son rápidamente absorbidas con un volumen de distribución de 300 litros, con amplia distribución tisular y cinética tricompartimental. ${ }^{13} \mathrm{Al}$ administrar por vía oral se presentan efectos hemodinámicos y ventilatorios mínimos con un volumen de administración pequeño. ${ }^{15}$

\section{Distribución}

Dexmedetomidina es un fármaco que se liga a proteínas plasmáticas en un $94 \%$ a la albúmina y a la a-1 glucoproteína. Muestra una amplia y rápida distribución corporal. En estudios experimentales en animales se detectó que cruza la barrera hematoencefálica y la placentaria. Usando un análisis no compartimental, la vida media de distribución fue cerca de 6 minutos en voluntarios saludables y se relacionó al peso corporal. El aparente volumen de distribución se ubicó en aproximadamente 1.31-2.46L/kg $(90-194 \mathrm{~L}) .{ }^{10} \mathrm{EI}$ modelo farmacocinético es el de un proceso de absorción bifásico, en donde se detecta un retraso entre el pico de concentración en el líquido cefalorraquídeo y el momento de la máxima reducción de la presión arterial que dura al menos 30 minutos. ${ }^{13}$

Metabolismo y Eliminación. La dexmedetomidina es eliminada principalmente a través de biotransformación por vía hepática. Menos del 1\% es excretada sin cambios; los metabolitos son excretados por vía renal (95\%) y fecalmente (4\%). La N-glucoronidación directa por uridina 5'- difosfo- glucoroniltransferasa (UGT2B10, UGT1A4) constituye cerca del $34 \%$ del metabolismo de la dexmedetomedina; la hidroxilación mediada por enzimas del citocromo P-450 principalmente CYP2A6 fue mostrado en microsomas hepáticos. Los isómeros $\mathrm{N}$-glucorónidos G-dex-1 (35\%) y G-dex2 (6\%), los O-glucorónidos de hidroxilados $\mathrm{N}$-methyl dexmedetomidina $(\mathrm{H}-1)(21 \%)$, y el producto de oxidación de imidazol H-3 (10\%). Estos metabolitos fueron considerados inactivos. La vida media de eliminación en voluntarios sanos es de $2.1-3.1 \mathrm{~h}$, sin embargo, en personas de unidad de cuidados intensivos oscila en el rango de 2.2 a $3.7 \mathrm{~h}$; el aclaramiento es aproximadamente de 0.6 a $0.7 \mathrm{~L} / \mathrm{min}$; valores rango de 0.51 a $0.89 \mathrm{~L} / \mathrm{min} .^{10,14,16}$

\section{Farmacodinamia}

La dexmedetomidina realiza sus efectos por activación de proteínas G (proteínas reguladoras fijadoras de nucleótidos de guanina). La activación de las proteínas $\mathrm{G}$ traduce una serie de procesos al modular la actividad celular. Los sucesos biológicos inician con la inhibición de la enzima adenilciclasa, al disminuir la concentración de 3'-5' adenosinmonofosfato cíclico (AMPc). El efecto subsecuente al finalizar el proceso es la estimulación de receptor $\alpha 2$, sin embargo, la sola baja concentración de AMPc no puede explicar algunos de los efectos fisiológicos presentados. Se han expresado una serie de mecanismos alternativos, entre los que destacan: la apertura de los canales de calcio dependientes de voltaje; el aumento del intercambio de sodio- hidrogeniones en el interior de las plaquetas; la apertura de diferentes canales de potasio hiperpolarizando la célula, lo que sugiere una manera de inhibir la actividad general neuronal. ${ }^{13}$

Para desarrollar su efecto hipnótico-sedante se disminuyen las concentraciones de GMPc cerebeloso; a nivel del locus ceruleus se une a los receptores $\alpha 2 a$ en donde ocasiona una disminución dosis-dependiente de la liberación de noradrenalina en la vía ascendente hacia la corteza cerebral, ocasionando disminución de la neurotransmisión noradrenérgica y serotoninérifa asociados a la transición de vigilia- sueño. ${ }^{10}$ 
Tiene una selectividad de 1600:1 por los receptores alfa 2 comparado con los receptores alfa 1 ; y con propiedades siete veces más selectivo cuando se compara con otro agonista alfa, la clonidina con una selectividad de 220:1. ${ }^{17}$ El mecanismo por el cual la dexmedetomidina puede incrementar la duración de un bloqueo nervioso periférico aún no es del todo claro, pero se cree que muy probablemente es un mecanismo perineural en lugar de un mecanismo sistémico o central. Se piensa que prolonga la duración mediante el bloqueo de la corriente catiónica. $^{18}$

La analgesia del bloqueo TAP con anestésicos locales solo dura unas pocas horas. ${ }^{19}$ La dexmedetomidina es un agonista a-2 adrenérgico que ha sido usado como un adyuvante para los anestésicos locales en el bloqueo TAP. Ocasiona una hiperpolarización prolongada nerviosa, así como un retraso en la restauración del potencial apoyado y previniendo la conducción de un nuevo potencial de acción. Este efecto es más pronunciado en las fibras desmielinizadas tipo $C$ que en las fibras tipo A; aunque la dexmedetomidina produce mejor analgesia que parálisis muscular, varios estudios han mostrado que la adición de dexmedetomidina a los anestésicos locales mejora la analgesia del bloqueo TAP. 20, 21, 22 Cuando se utiliza como complemento para el bloqueo nervioso periférico la dosis de dexmedetomidina es generalmente de $0.5-1 \mathrm{mcg} / \mathrm{kg}$ para lograr la prolongación deseada del anestésico local. ${ }^{23}$

Otros efectos asociados a dicho fármaco indican que favorece la diuresis a nivel renal vía disminución en la secreción de hormona antidiurética o por el bloqueo de su efecto en los túbulos renales. En ojos, disminuye la presión intraocular por la baja de humor acuoso mediante mecan ismos centrales y periféricos. ${ }^{13}$

A nivel gástrico: disminuye su secreción por estimulación directa de los receptores presináticos $\alpha-2$ en las células parietales y por inhibición vagal. En páncreas: existe tendencia a la hiperglicemia por disminución de la secreción de insulina. Cuenta con efectos antisialogógo por estimulación directa de adrenorreceptores alfa 2 y por inhibición de la liberación de acetilcolina. A nivel respiratorio se disminuye su frecuencia, aunque se mantiene la pendiente de la curva al $\mathrm{CO} 2$ durante la respuesta respiratoria. ${ }^{13}$

En sistema cardiovascular, la cardioprotección atribuida por la dexmedetomidina se considera manejada por la modulación del sistema nervioso autónomo; la disminución en la frecuencia cardíaca y la presión arterial cuando se utiliza, evita el desequilibrio entre aporte y demanda de oxígeno miocárdico ${ }^{23}$. Se ha observado también un aumento en la longitud del ciclo sinusal y tiempo de recuperación del nodo sinusal; la función del nodo auriculoventricular también se deprime lo que ocasiona que se prolongue el intervalo PR. ${ }^{24}$

\section{Efectos adversos}

Los efectos adversos más comunes de la dexmedetomidina se restringen hacia alteraciones relacionadas con depresión cardiovascular entre los que se incluyen: hipertensión al inicio de la administración, bradicardia, e hipotensión, así mismo puede presentarse bradicardia, fibrilación auricular e hipoxia con sedación dependiente de la dosis. La hipertensión, bradicardia e hipotensión, son propias de la activación del receptor $\alpha 2$ pre-y postsináptica, lo que ocasiona vasoconstricción, vasodilatación y bradicardia refleja. ${ }^{10}$ La hipertensión se produce mediante la estimulación de subtipos alfa de receptores en los músculos lisos vasculares. La hipertensión generalmente no requiere tratamiento y puede evitarse por la administración lenta u omisión de la dosis de carga. En relación al efecto perineural, la mayoría de los estudios reportan que en caso de manifestarse dichos efectos, han sido transitorios y reversibles. ${ }^{25}$

\section{Contraindicaciones}

No hay contraindicaciones absolutas para el uso de dexmedetomidina. Sin embargo, se debe utilizar con precau ción en pacientes con bradicardia e hipotensión, ya que el medicamento puede exacerbar estos síntomas. No hay requisitos específicos de monitoreo de medicamentos. Además, se sugiere que el nivel de sedación, frecuencia cardíaca, ritmo, presión arterial y oximetría de pulso debe controlarse estrechamente. ${ }^{25}$

\section{Toxicidad}

En la actualidad, no existe reversión o antídoto aprobado para la dexmedetomidina en humanos, sin embargo, el antagonista a2 selectivo atipamezol que se encuentra disponible en veterinarias ha revertido su efecto en modelos experimentales. ${ }^{25} \mathrm{La}$ atención de apoyo y la monitorización estrecha son los elementos básicos del tratamiento para la sobredosis.

\section{Conclusiones}

En concordancia con algunos autores y como resultado de la revisión de la literatura se concluye que la dexmedetomidina ha probado ser eficaz para prolongar la duración de los anestésicos locales en el bloqueo TAP hasta en un $40 \%{ }^{25}$

El uso combinado de Dexmedetomidina en el bloqueo TAP acorta el período de recuperación posquirúríg disminuyendo con ello la estancia intrahospitalaria y el 
riesgo potencial de infecciones intranosocomiales para los pacientes intervenidos.

Los efectos colaterales se observan disminuidos cuando el fármaco se utiliza con anestésicos locales de larga duración en el bloqueo TAP mejorando con ello la calidad de la analgesia de los pacientes postoperados. Además, se confirmó que la dosis propuesta de la dexmedetomidina oscila entre el 0.5 a $1 \mathrm{mcg}$ en bloqueos nerviosos periféricos.

A partir de estos hallazgos se postula que esta estrategia pudiera ser implementada con éxito en unidades médicas de atención ambulatoria, aumentando los indicadores de eficiencia en los procesos de esas unidades médicoquirú rgicas y en beneficio del paciente.

\section{Referencias}

[1] González-Esta villo, Ana, Jiménez-Ramos, Adriana, Rojas-Zarco, Eder, Velazco-Sordo, Chavéz-Ra mírez, Luis, Coronado-Ávila, Sergio (2018) "Correlación entre las escalas unidimensionales utilizadas en la medición de dolor postoperatorio" en Revista Mexicana de Anestesiología [En Línea]. Vol. 41, No. 1, pp. 7-14. Disponible en: https://www.medigraphic.com/cgi-

$\mathrm{bin} / \mathrm{new} /$ resumen.cgi?IDARTICULO=77264

[2] López, López, Vázquez (2017) Claves para Optimizar el tra tamiento del dolor agudo postopera torio domiciliario en cirugía a mbulatoria, Madrid, España: INSPIRA NETWORK.

[3] Gan, Habid, Miller, White, Apfelbaum (2014) "Incidence, patient satisfaction, and perceptions of postsurgical pain: Results from a US nationalsurvey" in Curr Med Res Opin [en Línea]. Vol. 30, No. 1, pp. 149 -160. Disponible en: doi: 10.1185/ 03007995.2013.860019

[4] Roger, Chou, et al (February de 2016) "Guidelines on the Management of Postoperative Pain" in The journal of Pain [en Línea]. No. 2, pp. 131 - 157. disponible en https://doi.org/10.1016/j.jpain.2015.12.008

[5] Suner, Kalayci, Sen, Kaya, Unver, Oguz (2019) "Postoperative analgesia after total abdominal hysterectomy: Is the transversus abdominisa plane block effective?" in Niger Journal Clinical Practice [en Línea]. Vol. 22, pp. 478-84. Disponible en http://www.njcponline.com/text.asp?2019/22/4/478/255938

[6] Akyol, Çiğdem, Özdilek, Aylin, Erbabacan, Emre, Özmumcu, Elif, Ekici1, Birsel, Köksall, Güniz, Altıntaş, Fatiş, Purisa, Sevim (2018). "Evaluation of the effects of subcostal transversus abdominis plane block on acute and subacute pain development following inguinal herniography: Randomized clinical study" in Agri [en Línea]. Vol. 30, No. 3, pp. 123-129. disponible en: doi: 10.5505/a gri.2018.49344

[7] Rudzik, Nadia, Ochoa, Marcos, Puca, Silvana, Dario, Raquel, Acosta, Cecilia (diciembre de 2016) "Bloqueos de la Pared Abdominalguiados por ultrasonido" en revista del hospital privedo de comunidad [en Línea]. Vol. 19, No. 1, pp. 8-15. Disponible en: https://www.hpc.org.ar/investiga cion/revista s/volumen-19/bloqueosde-la-pared-abdominal-guiados-por-ultra sonido/

[8] Chen, Qui, Xin, Liu, Yang, Bin (2018) "Addition of Dexmedetomidina or fentanyl to ropivacaine for transversus abdominis plane block: evaluation of effect on postoperative pain and quality of recovery in gynecological surgery" in Journal of Pain Research. No. 11, pp. 2879 2903. Disponible en doi: 10.2147 / JPR.S178516

[9] Alfonso, Joana, Reis, Flavio (Enero - Febrero de 2012) "Dexmedetomidina: Rol Actual en Anestesiología y Cuidados Intensivos" en Revista Brasileira de Anestesiología. Vol. 62, No. 1, pp. 118 -133. Disponible en: https://doi.org/10.1590/S003470942012000100015
[10] Weerink, Struys, Hannivort, Barends, Absalom, Colin (2017) "Clinica 1 Pharmacokinetics and Pharmacodynamics of Dexmedetomidine" in Clin Pharmacokinet. Vol. 56, No. 8, pp. 893-913. Disponible en doi: 10.1007 / s40262-017-0507-7

[11] Kleiman \& Jhonson (December de 2019) "Untapped Potential of Dexmedetomidina" in Anesthesia-analgesia. Vol. 129, Num. 6, pp. 1450-1453. Disponible en: DOI: 10.1213/ANE.0000000000004411

[12] Carrillo-Torres, Orlando, Pliego-Sánchez, María, Galleos-Allier, María, Santacruz-Martínez, Lourdes (2014). "Utilidad de la dexmedetomidina en diversos contextos en la medicina actual" en Revista Mexicana de Anestesiología. Vol. 37, No.1, pp. 27 - 34. Disponible en: https://www.medigraphic.com/cgi$\mathrm{bin} / \mathrm{new} /$ resumen.cgi?IDARTICULO=47859

[13] Haselman (2008) "Dexmedetomidine: a useful adjunct to consider in some high- risk situation" in Arthroscopy Association of North America. Vol. 76, Num. 5, pp. 335-339. Disponible en: https://www.ncbi.nlm.nih.gov/pubmed/18947160

[14] Yang, Tse, Lee, Joynt, Zuo (2019) "Large inter-individual variability in pharmacokinetics of dexmedetomidine and its two major $\mathrm{N}$ glucoronides in adult intensive care unit patients" Journal of Pharmaceutical and Biomedical Analysis. No. 175,pp. 1-9. Disponible en: doi: https://doi.org/10.1016/j.jpba.2019.07.025

[15] Alvaez, Ivone, Gallerdo, Leo, Martínez, Elvira, Mercado, Araceli, Gutiérrez, Abraham, Mendoza, Roberto, Arturo, Ana (2006) "Dexmedetomidina oral como medicación preanestésica en pacientes pediátricos. Comparación entre dosis y efectos clínicos" Anales Medicos. Vol. 51, No. 3, pp. 113-119. Disponible en: https://www.medigraphic.com/cgi-

bin/new/resumen.cgi?IDARTICULO=9899

[16] Abbot, L. (2019). Precedex ApprovalDocuments.

[17] Prabhakar, et al (2019) "Adjuvants in clinical regional anesthesia practice: A comprehensive review" in Best Practice and Research Clinical Anaesthesiology. Vol. 33, No. 4, pp. 415-423. Disponibe en: doi: $10.1016 /$ j.bpa.2019.06.001

[18] Kirskey, Haskins, Cheng, Liu (September de 2015) "Local Anesthetic Peripheral Nerve Block Adjuvants for Prolongation of Analgesia: Systematic Qualitative Review" in Plos One. Vol. 9, No.10, pp. 1-23. Disponible https:/www.ncbi.nlm.nih.gov/pmc/articles/PMC4565585/

[19] Bansal \& Garg (2019) "Effect of adding Dexmedetomidine to local Anesthetic agents for Transversus Abdominis Plane Block A meta analysis" in Clinical Journal of Pain. Vol. 35, No. 10, pp. 844 - 854. Disponible en: doi: 10.1097 / AJP.0000000000000742

[20] Ping, Ye, Wang, de Ye, Usted (2017) "Dexmedetomidine as an adjuvant to localanesthetics in brachialplexus blocks: a meta - analysis of randomized controlled trials" in Medicin. Vol. 96, No. 6, p. c5846. Disponible en doi: 10.1097 / MD.0000000000005846

[21] Almarakbi \& Kaki (2014) "Addition of dexmedetomidine to bupivacaine in transversus abdominis plane block potentiates postoperative pain relief among abdominal hysterectomy patients: A prospective randomized controlled trial" in Anaesth. Vol. 8, No. 2, pp. 161 - 166. Disponible en doi: 10.4103/1658-354X.130683

[22] Xu, Hu, Shen, McQuillan (2018) "Efficacy of US - guided transversus abdominis plane block and rectus sheath block with ropivacaine and dexmedetomidine in ederly high - risk patients" in Minerva Anestesiol . Vol. 84, No. 1, pp. 18-24. Disponible en: doi: 10.23736 / S03759393.17.11538-5

[23] Koyyalamud, Sen, Patil, Creel, Cornett, Fox, Kaye (2017) "Adjuvant agents in regional anesthesia in the ambulatory setting" in Curr Pain Headache Reports. Vol. 21, No. 1, p. 6. Disponible en: doi: 10.1007 / s11916-017-0604-1

[24] Hammer, et al (2008) "The effects of dexmedetomidine on cardiac electrophysiology in children" in Anesthesia Analgesia [en Línea]. Vol. 106, No. 1, pp. 79-83. Disponible en: doi: 10.1213 / 01.ane.0000297421.92857.4e 
[25] Rojas (2019) "Dexmedetomidina como coadyuvante en bloqueos de nervio periférico" en Revista de la Sociedad Española del Dolor [en Línea]. Vol. 26, No. 2, pp. 103-115. Disponible en: DOI: 10.20986/resed.2018.3695/2018 